\title{
Vitreous haemorrhage as a complication of HELLP syndrome
}

\author{
Steven R Leff, David L Yarian, Leo Masciulli, Stuart N Green, Roy E Baldomero
}

\begin{abstract}
In the last trimester of an uncomplicated pregnancy a woman developed a spontaneous vitreous haemorrhage. She was later diagnosed as having HELLP syndrome, a recently described disorder comprising haemolytic anaemia, elevated liver enzymes, and a low platelet count in women with severe preeclampsia or eclampsia. We believe this rare complication of pregnancy to be the cause of the intraocular bleeding.
\end{abstract}

Complications during pregnancy often affect the eyes. Sometimes the eye symptoms precede other systemic changes necessary for a correct diagnosis, so that ophthalmologists must be cautious when diagnosing a disorder in a pregnant woman with any new ocular condition.

\section{Case report}

A 33-year-old woman, seven months pregnant, was referred for ophthalmic examination. She had noted a sudden decline in the vision in her left eye several days earlier, not associated with any systemic complaint or undue exertion. Her past medical history was negative. She was unaware of any complications in her pregnancy. On ocular examination the Snellen visual acuity was 20/30 right eye, 20/100 left. The intraocular pressures and anterior segment examinations were normal in both eyes. A fundus examination of the right eye showed it to be entirely normal. A fundus examination of the left eye revealed two globular preretinal haemorrhages, each several disc diameters in size, straddling the supratemporal arcade. A small amount of breakthrough vitreous haemorrhage obscured much of the detail of the posterior pole findings, though the retina was otherwise grossly intact and appeared normal. The patient's obstetrician was advised of the findings, although the cause of this condition was not clear. A follow-up obstetric examination and blood pressure determinations were reported as normal.

Over the next two weeks the vitreous haemorrhage gradually resolved, though there was still a preretinal haemorrhage in the superior fundus, presumably overlying the source of the inital bleed. Approximately one month after the initial ophthalmic examination the patient developed uncontrolled hypertension as a result of toxaemia and was referred to a tertiary care centre for further obstetric management. An emergency caesarean section was required. Laboratory work up at that time revealed a haemoglobin of $14.3 \mathrm{~g} / \mathrm{dl}$, a platelet count of $28 \times 10^{\circ} / 1$, serum aspartate transaminase (SGOT) of 365 units, serum glutamic pyruvic transaminase (SGPT) of 222 units, and lactic dehydrogenase (LDH) of 1217 units. Although anaemia was not present, the remaining abnormal laboratory values supported a diagnosis of HELLP syndrome (haemolytic anaemia, elevated liver enzymes, and a low platelet count in women with severe pre-eclampsia or eclampsia).

The patient's metabolic parameters gradually returned to normal, and both mother and child did well. The patient returned for follow-up ophthalmic examination one month after delivery. The Snellen visual acuity in the left eye was then 20/30. Fundus examination of the left eye showed that nearly all the preretinal haemorrhage had become reabsorbed. A tiny fibrotic scar was noted over a small branch arteriole in the superior fundus, which was previously obscured by the overlying blood, and which probably represented the healed focus of the previous haemorrhage.

\section{Discussion}

Ocular changes associated with toxaemia complicating pregnancy have been described elsewhere. ${ }^{1}$ In the HELLP syndrome, ${ }^{2}$ observed in women with severe pre-eclampsia or eclampsia, severe hypertension is often only transient or entirely absent. We believe that our patient suffered from unrecognised thrombocytopenia secondary to HELLP syndrome at the time of her initial visual complaint. Although the retinal haemorrhage was probably a result of this clotting abnormality, an unrecognised transient episode of hypertension related to pre-eclampsia may have also contributed to the rupture of the susceptible site in a peripheral retinal vessel. To our knowledge this is the first description of a patient suffering an intraocular haemorrhage as a consequence of HELLP syndrome.

\footnotetext{
1 Sunness JS. The pregnant woman's eye. Surv Ophthalmol 1988; 32: $219-44$.

2 Weinstein L. Syndrome of hemolysis, elevated liver enzymes and low platelet count: a severe consequence of hypertension in pregnancy. Am F Obstet Gynecol 1982;142: 159-63.
} 